\title{
ON THE ADO THEOREM FOR FINITE LIE CONFORMAL ALGEBRAS WITH LEVI DECOMPOSITION
}

\author{
PAVEL KOLESNIKOV
}

\begin{abstract}
We prove that a finite torsion-free conformal Lie algebra with a splitting solvable radical has a finite faithful conformal representation.
\end{abstract}

\section{INTRODUCTION}

Conformal algebras were introduced in [10 as an algebraic formalism describing properties of the singular part of the operator product expansion (OPE) in conformal field theory (i.e., conformal Lie algebra is a singular part of a vertex operator algebra). The same notion is often called vertex Lie algebra (see, e.g., [8]). The relations of conformal algebra with other areas of mathematics include hamiltonian formalism in the theory of evolution equations [1, variational calculus [7, and the theory of algebraic structures arising from algebraic topology and K-theory [13], see [1].

From the formal point of view, the category of conformal algebras is the first member (after "ordinary" algebras over a field) in the hierarchy of pseudo-algebras over cocommutative bialgebras, as described in 11. The case of ordinary algebras corresponds to the one-dimensional bialgebra $H=\mathbb{k}$, conformal algebras are modules over the polynomial bialgebra $H=\mathbb{k}[\partial]$. As in the case of ordinary algebras, an associative conformal algebra turns into a Lie conformal algebra by means of the standard morphism of the corresponding operads given by $x_{1} x_{2} \mapsto x_{1} x_{2}-x_{2} x_{1}$. The converse statement does not hold in general: There exist Lie conformal algebras that cannot be embedded into an associative one [16]. However, it is an open problem whether a finite (i.e., finitely generated over $H$ ) conformal Lie algebra has such an embedding. A more precise problem can be stated as follows: Whether a finite conformal Lie algebra which is torsion-free as an $H$-module can be faithfully represented by conformal endomorphisms [10] of a finitely generated torsion-free $H$-module $M$ ? This statement would be the "conformal analogue" of the classical Ado Theorem. The purpose of this paper is to make a step towards the solution of this problem.

The Ado Theorem is a fundamental fact in the theory of Lie algebras, however, it has a reputation of "strange" theorem [14. Every known proof of this statement (e.g., the classical ones [9], 15], or the short and elegant proof in [14]) exploits the following basic properties of finite-dimensional Lie algebras:

(A1) The Poincaré - Birkhoff-Witt (PBW) Theorem (at least for nilpotent algebras);

Partially supported by RFBR (project 12.01.00329), FAPESP 2012/04704-0, and the Federal Target Program (contract 14.740.11.0346). 
(A2) Complete reducibility of finite-dimensional modules over semisimple algebras;

(A3) The image of a solvable Lie algebra under its derivation is nilpotent;

(A4) The Levi Theorem (splitting of the solvable radical).

For conformal algebras (even for finite ones), these properties do not hold in general. This is the reason why proving an analogue of the Ado Theorem for conformal algebras is a challenging problem.

In [17, it was shown that a nilpotent conformal Lie algebra embeds into a nilpotent associative conformal algebra, thus, the property (A1) is not essential for our purpose.

In [12, we eluded the property (A2): The existence of a finite faithful representation was proved for torsion-free finite Lie conformal algebras of "classical type", i.e., with a splitting solvable radical and without Virasoro elements in the semisimple part. These conformal algebras turn to be subalgebras of current conformal algebras over finite-dimensional ordinary Lie algebras. It is interesting to note that the proof in [12] was not based on the Ado Theorem for ordinary algebras. Therefore, the problem was solved for those conformal Lie algebras satisfying the analogues of (A3) and (A4).

The purpose of this note is to eliminate (A3). Namely, we prove the conformal version of the Ado Theorem for an arbitrary finite torsion-free conformal Lie algebra $L$ with a splitting solvable radical.

Let $\mathbb{k}$ be a field of zero characteristic. Without loss of generality (see 12, Lemma 7]) we may assume $\mathbb{k}$ to be algebraically closed. Throughout the paper, the symbol $\otimes$ without a subscript means the tensor product of spaces over $\mathbb{k}$.

\section{Conformal algebras and their Representations}

A conformal algebra 10 is a unital module $C$ over the polynomial algebra $H=$ $\mathbb{k}[\partial]$ equipped with a polynomial-valued $\mathbb{k}$-linear operation $(\cdot(\lambda)):. C \otimes C \rightarrow C[\lambda]$, where $\lambda$ is a formal variable (this operation is called $\lambda$-product), satisfying the following axioms:

$$
\left(\partial a_{(\lambda)} b\right)=-\lambda\left(a_{(\lambda)} b\right), \quad\left(a_{(\lambda)} \partial b\right)=(\lambda+\partial)\left(a_{(\lambda)} b\right) .
$$

Conformal algebra is said to be finite if it is finitely generated as a module over $H$.

Equivalently, one may consider a conformal algebra $C$ as a linear space over $\mathbb{k}$ with one linear operation $\partial$ and with an infinite family of bilinear products $(\cdot(\alpha) \cdot)$, $\alpha \in \mathbb{k}$, where $\left(a_{(\alpha)} b\right)=\left.\left(a_{(\lambda)} b\right)\right|_{\lambda=\alpha}$ for $a, b \in C$.

Another interpretation of a conformal algebra structure is based on the notion of a pseudo-tensor category. Let us sketch the ideas developed in [1] to clarify the relations between "ordinary" and conformal algebras and their representations.

Consider the polynomial algebra $H=\mathbb{k}[\partial]$ and denote by $\mathcal{M}(H)$ the class of (left) unital $H$-modules. Recall that $H$ carries the standard bialgebra structure given by coproduct $\Delta(\partial)=\partial \otimes 1+1 \otimes \partial$ and counit $\varepsilon(\partial)=0$. Given $M_{1}, \ldots, M_{n}, M \in \mathcal{M}$, denote

$$
P_{n}\left(M_{1}, \ldots, M_{n} ; M\right)=\operatorname{Hom}_{H \otimes n}\left(M_{1} \otimes \cdots \otimes M_{n}, H^{\otimes n} \otimes_{H} M\right),
$$

where $H^{\otimes n}$ is considered as the outer product of regular right $H$-modules. For the class $\mathcal{M}(H)$, the spaces (2) play the same role as the spaces of polylinear maps for the class of linear spaces over a field. There exist a composition rule and an 
equivariant symmetric group action on (2) such that $\mathcal{M}(H)$ turns into a pseudotensor category in the sense of [3]. Conformal algebra $C$ is exactly the same as an algebra in $\mathcal{M}(H)$ with one binary operation (pseudo-product) $\mu \in P_{2}(C, C ; C)$. Namely, for $a, b \in C$,

$$
\left(a_{(\lambda)} b\right)=\sum_{n \geq 0} \lambda^{n} c_{n}, \quad c_{n} \in C,
$$

we have

$$
\mu(a, b)=\sum_{n \geq 0}\left((-\partial)^{n} \otimes 1\right) \otimes_{H} c_{n} .
$$

In these terms, (10) is equivalent to $H^{\otimes 2}$-linearity of $\mu$.

These data are enough to define what is an algebra (associative, commutative, Lie, etc.), a homomorphism, a representation (module), and a cohomology in the class $\mathcal{M}(H)$ [1.

For example, the associativity of a conformal algebra $C$ may be expressed in terms of pseudo-product as

$$
\mu(\mu, \mathrm{id})=\mu(\mathrm{id}, \mu) \in P_{3}(C, C, C ; C),
$$

and in terms of $\lambda$-product as

$$
\left(a_{(\alpha)}\left(b_{(\beta)} c\right)\right)=\left(\left(a_{(\alpha)} b\right)_{(\alpha+\beta)} c\right), \quad a, b, c \in C, \alpha, \beta \in \mathbb{k} .
$$

Similarly, the anti-commutativity and the Jacobi identity for a pseudo-product $\mu$ have the same form as for "ordinary" product:

$$
\begin{gathered}
\mu=-\mu^{(12)}, \\
\mu(\mathrm{id}, \mu)-\mu(\mathrm{id}, \mu)^{(12)}=\mu(\mu, \mathrm{id}) .
\end{gathered}
$$

In terms of $\lambda$-product, these relations turn into

$$
\begin{gathered}
\left(a_{(\alpha)} b\right)=-\left(b_{(-\partial-\alpha)} a\right), \\
\left(a_{(\alpha)}\left(b_{(\beta)} c\right)\right)-\left(b_{(\beta)}\left(a_{(\alpha)} c\right)\right)=\left(\left(a_{(\alpha)} b\right)_{(\alpha+\beta)} c\right),
\end{gathered}
$$

for $a, b, c \in C, \alpha, \beta \in \mathbb{k}$, respectively.

As in the case of ordinary algebras, an associative conformal algebra $C$ with respect to the new $\lambda$-product

$$
\left[a_{(\alpha)} b\right]=\left(a_{(\alpha)} b\right)-\left(b_{(-\partial-\alpha)} a\right)
$$

satisfies anti-commutativity and Jacobi identity, i.e., is a Lie conformal algebra. Below we will use the brackets $\left[\cdot(\lambda)^{\cdot}\right]$ to denote $\lambda$-products in all Lie conformal algebras.

The notions of an ideal, solvability, and nilpotence have the natural interpretation in conformal algebras. A conformal algebra $C$ is called trivial if $C_{(\alpha)} C=0$ for all $\alpha \in \mathbb{k}$. A nontrivial conformal algebra is simple if it has no nonzero proper ideals. If $C$ has no nonzero solvable ideals then it is said to be semisimple.

To define a conformal algebra structure on an $H$-module, it is enough to define the $\lambda$-product on its generators over $H$ and then expand it to the entire module by means of (11).

Example 1. Let $\mathfrak{a}$ be an ordinary algebra over $\mathbb{k}$. Then the free $H$-module $H \otimes \mathfrak{a}$ can be considered as a conformal algebra with respect to operation

$$
a_{(\lambda)} b=a b, \quad a, b \in \mathfrak{a},
$$


called current conformal algebra Cur $\mathfrak{a}$. If $\mathfrak{a}$ is associative (Lie, alternative, etc) then so is Cur a.

Example 2. The free $H$-module of rank one generated by an element $v$ turns into a Lie conformal algebra by means of the operation

$$
\left[v_{(\lambda)} v\right]=(\partial+2 \lambda) v
$$

This structure is called Virasoro conformal algebra Vir.

Example 3. Suppose $\mathfrak{g}$ is a Lie algebra. The direct sum of $H$-modules $H v \oplus(H \otimes \mathfrak{g})$ with respect to

$$
\begin{gathered}
{\left[v_{(\lambda)} v\right]=(\partial+2 \lambda) v, \quad\left[v_{(\lambda)} a\right]=(\partial+\lambda) a, a \in \mathfrak{g}} \\
{\left[a_{(\lambda)} b\right]=[a, b], a, b \in \mathfrak{g},}
\end{gathered}
$$

is a Lie conformal algebra denoted by $\operatorname{Vir} \ltimes \operatorname{Cur} \mathfrak{g}$, the semi-direct product of conformal algebras.

The classification of finite conformal algebras is given by the following

Theorem 1 ([6]). (i) A simple finite Lie conformal algebra is isomorphic either to Vir or to Cur $\mathfrak{g}$, where $\mathfrak{g}$ is a finite-dimensional simple Lie algebra.

(ii) A semi-simple finite Lie conformal algebra is a direct sum of conformal algebras $C_{1} \oplus \cdots \oplus C_{k}$, where each $C_{i}$ is either simple or isomorphic to Vir $\ltimes$ Cur $\mathfrak{g}$, $\mathfrak{g}$ is a simple finite-dimensional Lie algebra.

An arbitrary finite associative or Lie conformal algebra $C$ obviously has a maximal solvable ideal (radical) $R$ such that $C / R$ is a semisimple conformal algebra. However, for Lie conformal algebras there is no analogue of the Levi Theorem stating $C \simeq(C / R) \ltimes R$. As in the case of ordinary Lie algebras, the abelian extensions of conformal algebras are described in terms of the second cohomology group. The corresponding notions were introduced in [2, were cohomologies of simple and semisimple finite conformal algebras with coefficients in their irreducible modules were computed; many of them are nontrivial.

Suppose $C$ is an associative (Lie) conformal algebra. Then a conformal module over $C$ is an $H$-module $M \in \mathcal{M}(H)$ equipped with $\nu \in P_{2}(C, M ; M)$ satisfying the appropriate associativity (Jacobi) identity. It terms of $\lambda$-product these notions were introduced and studied in [4]

Remark 1. In this study, we consider those conformal algebras (and their modules) that are torsion-free as $H$-modules. The reason for such a restriction comes from the following observation [6, 1]. If $U, V, W \in \mathcal{M}(H), \mu \in P_{2}(U, V ; W)$, then $\mu(a, V)=$ $\mu(U, b)=0$ for every torsion elements $a \in U, b \in V$. Hence, a conformal algebra with a nonzero $H$-torsion has no faithful representations.

Irreducible representations of finite simple and semisimple Lie conformal algebras are described by

Theorem 2 (4]). (i) A finite irreducible conformal module over Vir is a free $H$ module of rank one generated by an element $u$ such that

$$
v_{(\lambda)} u=(\alpha+\partial+\Delta \lambda) u, \quad \alpha, \Delta \in \mathbb{k}, \Delta \neq 0 .
$$

Such a module is denoted $M_{\alpha, \Delta}$. 
(ii) Suppose $\mathfrak{g}$ is a finite-dimensional simple Lie algebra. A finite irreducible conformal module over Cur $\mathfrak{g}$ is isomorphic to $H \otimes U$, where $U$ is a finite-dimensional irreducible $\mathfrak{g}$-module, and

$$
g_{(\lambda)} u=g u, \quad g \in \mathfrak{g}, u \in U .
$$

This module is natural to denote by $\operatorname{Cur} U$.

(iii) Suppose $\mathfrak{g}$ is a finite-dimensional simple Lie algebra. A finite conformal module $M_{\alpha, \Delta, U}$ over $\operatorname{Vir} \ltimes \operatorname{Cur} \mathfrak{g}$ is constructed as $H \otimes U$, where $U$ is a finitedimensional $\mathfrak{g}$-module, and

$$
\begin{gathered}
v_{(\lambda)} u=(\alpha+\partial+\Delta \lambda) u, \quad \alpha, \Delta \in \mathbb{k}, \\
g_{(\lambda)} u=g u, \quad g \in \mathfrak{g}, u \in U .
\end{gathered}
$$

Every finite irreducible conformal module over $\operatorname{Vir} \ltimes \operatorname{Cur} \mathfrak{g}$ is isomorphic to $M_{\alpha, \Delta, U}$, where either $U$ is an irreducible $\mathfrak{g}$-module (and $\Delta$ is an arbitrary scalar) or $U$ is trivial one-dimensional and $\Delta \neq 0$.

If $L$ is a Lie conformal algebra and $a \in L$ then the operation $D_{\lambda}=\left[a_{(\lambda)} \cdot\right]: L \rightarrow$ $L[\lambda]$ has the following property: $D_{\lambda}\left(\left[x_{(\mu)} y\right]\right)=\left[\left(D_{\lambda} x\right)_{(\lambda+\mu)} y\right]+\left[x_{(\mu)}\left(D_{\lambda} y\right)\right]$ for all $x, y \in L$. In general, such a map $D_{\lambda}$ is called a conformal derivation of $L$.

Lemma 1 (c.f. 4, Proposition 3.1]). Assume $C$ is a conformal algebra which is a conformal module over $\mathrm{Vir}=H v$ such that $D_{\lambda}=\left(v_{\lambda} \cdot\right)$ is a conformal derivation of $C$. Suppose $M$ is a conformal Vir-submodule of $C$ isomorphic to $M_{\alpha, \Delta}$ for some $\alpha, \Delta \in \mathbb{k}$, and $N$ is a trivial conformal Vir-submodule of $C$. Then $\left(N_{(\lambda)} M\right)=0$ in $C$.

Proof. Denote $M=H u$, where $\left(v_{(\lambda)} u\right)=(\alpha+\partial+\lambda \Delta) u$. Choose an arbitrary $a \in N$ and consider $a_{(\lambda)} u=\sum_{i} \lambda^{i} c_{i} \in C[\lambda]$. Then

$$
D_{0}\left(a_{(\lambda)} u\right)=a_{(\lambda)} D_{0} u=(\alpha+\lambda+\partial)\left(a_{\lambda} u\right) .
$$

Since $D_{0}\left(\sum_{i} \lambda^{i} c_{i}\right)=\sum_{i} \lambda^{i} D_{0} c_{i}$, the degree in $\lambda$ of the left-hand side of (3) does not exceed the degree of $\left(a_{(\lambda)} u\right)$. If the latter is finite then the right-hand side of (3) has a greater degree than $\left(a_{(\lambda)} u\right)$. The contradiction obtained proves $\left(a_{(\lambda)} u\right)=0$.

\section{COMPOSITION SERIES IN CONFORMAL MODUlES}

Note that a finite conformal module even over a finite simple Lie conformal algebra cannot (in general) be decomposed into a direct sum of irreducible ones (see [5] for a systematic study of extensions). Moreover, although the lattice of conformal submodules in a given module is modular (Dedekind), a finite composition series may not exist even in a finite conformal module. As an example, consider the free $H$-module $M_{1}$ of rank one with respect to a trivial action of a conformal algebra (say, over Vir). Then there exists a normal series

$$
0 \subset \partial^{n} M_{1} \subset \partial^{n-1} M_{1} \subset \cdots \subset \partial M \subset M
$$

of arbitrary length $n$. However, we may still apply a kind of triangular decomposition to those conformal modules we need (see Lemma 4 below).

Some of the results of this section can be recovered from [4, 5, but we state their proofs for readers' convenience. 
Lemma 2. Let $L$ be a conformal algebra of type Vir $\ltimes$ Cur $\mathfrak{g}$, where $\mathfrak{g}$ is either a finite-dimensional simple Lie algebra or $\mathfrak{g}=0$ (i.e., $L=$ Vir). Suppose $V, M$, and $E$ are three finite conformal modules over $L$ such that $M$ is irreducible, $V$ is a trivial torsion-free L-module, and there exists a short exact sequence

$$
0 \rightarrow V \rightarrow E \rightarrow M \rightarrow 0
$$

of conformal modules over $L$. Then $E \simeq V \oplus M$, the direct sum of conformal modules over $L$.

Proof. Extensions of conformal modules may be described via the corresponding conformal cocycles (see [5, 7]). Let $L, M$, and $V$ be as in the statement. A $\mathbb{k}$-linear map $\varphi_{\lambda}: L \otimes M \rightarrow V[\lambda]$ satisfying the $3 / 2$-linearity condition similar to (1) is called a cochain. If a cochain $\varphi_{\lambda}$ satisfies

$$
\varphi_{\lambda}\left(a, b_{(\mu)} u\right)-\varphi_{\mu}\left(b, a_{(\lambda)} u\right)=\varphi_{\lambda+\mu}\left(\left[a_{(\lambda)} b\right], u\right)
$$

for all $a, b \in L, u \in M$ then $\varphi_{\lambda}$ is said to be a cocycle. For every $H$-linear map $\tau: M \rightarrow V$ its differential $\delta_{\lambda} \tau: L \otimes M \rightarrow V$ defined by $\left(\delta_{\lambda} \tau\right)(a, u)=\tau\left(a_{(\lambda)} u\right)$ is a cocycle.

Obviously, every cocycle $\varphi_{\lambda}: L \otimes M \rightarrow V[\lambda]$ gives rise to a conformal $L$-module $E=E(M, V, \varphi)$ such that the sequence (41) is exact. Conversely, every exact sequence (4) allows to define the corresponding cocycle [7, Theorem 2.1]. Moreover, for given cocycles $\varphi_{\lambda}$ and $\psi_{\lambda}$, the extensions $E(M, V, \varphi)$ and $E(M, V, \psi)$ are isomorphic if and only if $\varphi_{\lambda}-\psi_{\lambda}=\delta_{\lambda} \tau$ for an appropriate $\tau: M \rightarrow V$.

To prove the statement, it is enough to show that every cocycle $\varphi_{\lambda}: L \otimes M \rightarrow V$ is equal to $\delta_{\lambda} \tau$ for some $\tau: M \rightarrow V$. By Theorem 2 there are three cases to be considered.

Case 1: $L=$ Vir, $M=M_{\alpha, \Delta}, \Delta \neq 0$;

Case 2: $L=\operatorname{Vir} \ltimes \operatorname{Cur} \mathfrak{g}, M=M_{\alpha, \Delta, U}, \Delta \neq 0$;

Case 3: $L=\operatorname{Vir} \ltimes \operatorname{Cur} \mathfrak{g}, M=M_{\alpha, 0, U}, U$ is a nontrivial $\mathfrak{g}$-module.

Let us consider Case 2 and Case 3 in details since Case 1 is completely covered by calculations from Case 2 .

In Case 2, suppose $\varphi_{\lambda}(v, u)=\sum_{i} f_{i}(\partial, \lambda) w_{i}(u), u \in U$, where $f_{i}(\partial, \lambda) \in \mathbb{k}[\partial, \lambda]$, $w_{i}(u)$ are linearly independent over $\mathbb{k}$ in $V$. Then by (5) we have (for $a=b=v$, $\mu=0)$

$$
(\alpha+\partial) f_{i}(\partial, \lambda)=(\alpha+\Delta \lambda+\partial) f_{i}(\partial, 0) .
$$

Since $\Delta \neq 0, \alpha+\partial$ divides $f_{i}(\partial, 0)$, so $f_{i}(\partial, \lambda)=(\alpha+\Delta \lambda+\partial) h_{i}(\partial)$. Define $\tau(u)=\sum_{i} h_{i}(\partial) w_{i}(u) \in V$. Then $\varphi_{\lambda}(v, u)=\tau\left(v_{(\lambda)} u\right)=\left(\delta_{\lambda} \tau\right)(v, u)$. Therefore, without loss of generality (replacing $\varphi_{\lambda}$ with $\left.\varphi_{\lambda}-\delta_{\lambda} \tau\right)$ we may assume $\varphi_{\lambda}(v, u)=0$.

Now, consider $g \in \mathfrak{g}$. Then by (5) we have (for $a=v, b=g, \lambda=0$ )

$$
\varphi_{0}(v, g u)-\varphi_{\mu}(g,(\partial+\alpha) u)=\varphi_{\mu}(\partial g, u),
$$

that implies $(\alpha+\partial) \varphi_{\mu}(g, u)=0$. Since $V$ is torsion-free, we have $\varphi_{\lambda} \equiv 0$.

In Case 3, the same computations with $a=b=v$ imply that $\varphi_{\lambda}(v, u)$ do not depend on $\lambda$ for every $u \in U$. Suppose $V=H \otimes W,\left\{w_{i}\right\}_{i \in I}$ is a basis of the linear space $W$. Then for every $u \in U$ we may write $\varphi_{\lambda}(v, u)=\sum_{i} f_{i}^{u}(\partial) w_{i}, f_{i}^{u} \in H$. Let us represent $f_{i}^{u}(\partial)$ as $(\alpha+\partial) h_{i}^{u}(\partial)+\beta_{i}^{u}, \beta_{i}^{u} \in \mathbb{k}$. Then for $\tau(u)=\sum_{i} h_{i}^{u}(\partial) w_{i}$ we have $\left(\delta_{\lambda} \tau\right)(v, u)=\varphi_{\lambda}(v, u)-\sum_{i} \beta_{i}^{u} w_{i}$. Hence, we may assume without loss of generality that $f_{i}^{u}(\partial)$ are constants from $\mathbb{k}$. 
Suppose $\varphi_{\lambda}(g, u)=\sum_{i} h_{i}^{g, u}(\partial, \lambda) w_{i}, h_{i}^{g, u} \in \mathbb{k}[\partial, \lambda]$. By the same reasons as in Case 2 we have

$$
\varphi_{0}(v, g u)=(\alpha+\partial) \varphi_{\mu}(g, u)
$$

so $f_{i}^{g u}=(\alpha+\partial) h_{i}^{g, u}(\partial, \mu)$. Since the left-hand side is constant, we obtain $f_{i}^{g u}=$ $h_{i}^{g, u}=0$ for all $g \in \mathfrak{g}, u \in U$. Hence, $\varphi_{\lambda} \equiv 0$.

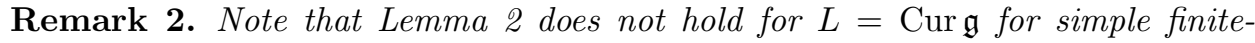
dimensional Lie algebras $\mathfrak{g}$, see [5, Proposition 4.4].

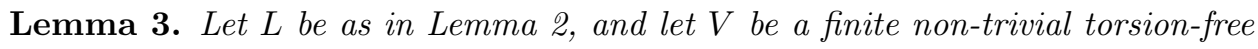
conformal module over $L$. Then $V$ contains an irreducible conformal submodule.

Proof. Choose a nontrivial conformal $L$-submodule $W$ of minimal rank in $V$. Denote by $\mathcal{R}$ the set of all those nontrivial conformal $L$-submodules in $W$ that have the same rank over $H$ as $W$. For every $U \in \mathcal{R}$ there exists $h \in H, h \neq 0$, such that $h W \subseteq U$ (since $W / U$ coincides with its torsion). Denote

$$
V_{0}=\bigcap_{U \in \mathcal{R}} U
$$

then $L_{(\lambda)} W \subseteq V_{0}[\lambda]$. Since $L$ is a perfect algebra $\left(\sum_{\alpha \in \mathbb{k}}\left[L_{(\alpha)} L\right]=L\right)$, the last expression implies that $V_{0}$ is a nontrivial conformal $L$-module. Hence, the rank of $V_{0}$ coincides with the rank of $W$, and there are no nontrivial proper conformal $L$-submodules in $V_{0}$.

Choose a maximal (proper) conformal $L$-submodule $U_{0}$ in $V_{0}$. By the construction of $V_{0}, U_{0}$ has to be trivial. The maximality of $U_{0}$ implies $M_{0}=V_{0} / U_{0}$ to be an irreducible conformal $L$-module. By Lemma 2 there exists a conformal $L$ submodule in $V_{0}$ isomorphic to $M_{0}$. This is the desired submodule.

Lemma 4. Let $L$ be as in Lemma Q, and let $M$ be a finite conformal module over $L$. Then there exists a chain of submodules

$$
0=M_{-1} \subset M_{0} \subset \cdots \subset M_{n}=M,
$$

where $M_{k} / M_{k-1}$ is either irreducible or trivial torsion-free or coincides with its torsion (hence, trivial).

Proof. This is an immediate corollary of Lemma 3. Note that if $M$ is nontrivial torsion-free conformal $L$-module then $M_{0}$ has to be irreducible.

Lemma 5. Assume $C$ is a torsion-free conformal algebra which is a conformal module over $\mathrm{Vir}=H v$ such that $D_{\lambda}=\left(v_{\lambda} \cdot\right)$ is a conformal derivation of $C$. Suppose $M$ is a conformal Vir-submodule of $C$ isomorphic to $M_{\alpha, \Delta}$ for some $\alpha, \Delta \in \mathbb{k}$, and $N$ is an arbitrary finite conformal Vir-submodule of $C$. If $\left(N_{(\lambda)} M\right)$ falls into the kernel of $D_{0}$ then $\left(N_{(\lambda)} M\right)=0$ in $C$.

Proof. Let $M=H u, D_{0} u=(\alpha+\partial) u$. Consider the triangular decomposition from Lemma 4 for $N$ :

$$
0=N_{-1} \subset N_{0} \subset \cdots \subset N_{r}=N .
$$

Assume $k \geq 0$ is the minimal index such that $\left(N_{k(\lambda)} M\right) \neq 0$.

If $N_{k} / N_{k-1}$ is a trivial Vir-module then for every $a \in N_{k}$ we have $D_{0} a \in N_{k-1}$, so

Since $C$ is torsion-free, $\left(a_{(\lambda)} u\right)=0$.

$$
0=D_{0}\left(a_{(\lambda)} u\right)=a_{(\lambda)} D_{0} u=(\alpha+\partial+\lambda)\left(a_{(\lambda)} u\right) .
$$


If $N_{k} / N_{k-1}$ is an irreducible Vir-module isomorphic to $M_{\beta, \delta}$ then $N_{k}=\mathrm{Ha}+$ $N_{k-1}$, where $D_{0} a \in(\beta+\partial) a+N_{k-1}$. In this case,

$$
0=D_{0}\left(a_{(\lambda)} u\right)=\left(D_{0} a_{(\lambda)} u\right)+a_{(\lambda)} D_{0} u=(\beta+\alpha+\partial)\left(a_{(\lambda)} u\right),
$$

so $a_{(\lambda)} u=0$.

\section{Finite faithful Representation}

Suppose $L$ is a finite torsion-free conformal Lie algebra with the maximal solvable ideal $R$. Assume $R$ splits in $L$, i.e., $L=L_{0} \ltimes R$, where $L_{0}$ is semisimple. Denote by $Z(L)$ the center of $L$.

First, consider the case when $R$ is nilpotent.

Proposition 1. If $R$ is nilpotent and $L_{0}$ contains a summand $L_{1}$ isomorphic either to Vir or to Vir $\ltimes$ Cur $\mathfrak{g}$ ( $\mathfrak{g}$ is a simple finite-dimensional Lie algebra) such that $R$ is a nontrivial conformal $L_{1}$-module then $L$ contains a nonzero ideal $I$ such that $I \cap Z(L)=0$ and $\left[R_{(\lambda)} I\right]=0$.

Proof. Consider the sequence of ideals

$$
R=R^{1} \supset R^{2} \supset \cdots \supset R^{n-1} \supset R^{n}=0,
$$

where $R^{l+1}=\sum_{\alpha \in \mathbb{k}}\left[R_{(\alpha)} R^{l}\right]$. Choose the maximal $m$ such that $R^{m}$ is a nontrivial $L_{1}$-module. By Lemma 3 there exists an irreducible conformal $L_{1}$-submodule $M_{0}$ in $R^{m}$.

It turns out that $M_{0}$ is the desired ideal in $L$. Let us consider in details the case when $L_{1}=\operatorname{Vir} \ltimes \operatorname{Cur} \mathfrak{g}$ and $M_{0}=M_{\alpha, \Delta, U}$ as in Theorem 2(iii). By $v$ we denote the canonical Virasoro element of $L_{1}$. It is enough to show that $\left[L_{k(\lambda)} M_{0}\right] \subseteq M_{0}[\lambda]$ for all $k>1$ and $\left[R_{(\lambda)} M_{0}\right] \subseteq M_{0}[\lambda]$.

Let $u \in U$. Then $H u$ is a conformal Vir-submodule in $L$ which is isomorphic to $M_{\alpha, \Delta}$. For $k>1$, the summand $L_{k}$ is a trivial Vir-module. Hence, by Lemma 1 we have $\left[L_{k(\lambda)} M_{0}\right]=0$.

Finally, note that $\left[R_{(\lambda)} M_{0}\right] \subseteq R^{m+1}[\lambda]$. By the choice of $m, R^{m+1}$ is a trivial $L_{1}$-module. Therefore, we may apply Lemma 5 to conclude $\left[R_{\lambda} M_{0}\right]=0$.

We have found an ideal $I=M_{0} \neq 0$ in $L$ which has zero intersection with the center $Z$ of $L$ since $\left[v_{(0)} \cdot\right]$ has no kernel in $M_{0}$.

Now, let us expand the results of Proposition 1 to the more general case.

The conformal version of the Lie theorem for solvable Lie conformal algebras [6 implies, in particular, that $R^{\prime}=R^{2}$ is nilpotent.

Proposition 2. If $L_{0}$ contains a summand $L_{1}$ isomorphic either to Vir or to $\operatorname{Vir} \ltimes \operatorname{Cur} \mathfrak{g}(\mathfrak{g}$ is a simple finite-dimensional Lie algebra) such that $R$ is a nontrivial conformal $L_{1}$-module then $L$ contains a nonzero ideal $I$ such that $I \cap Z(L)=0$.

Proof. Case 1. Assume $R^{\prime}$ is a trivial $L_{1}$-module. By Lemma 3, $R$ contains an irreducible conformal $L_{1}$-submodule $M_{0}=M_{\alpha, \Delta, U}$. For each $0 \neq u \in U, H u$ is a conformal module over Vir isomorphic to $M_{\alpha, \Delta}$. Then $\left[L_{i(\lambda)} H u\right]=0$ by Lemma 1. Moreover, $\left[R_{(\lambda)} H u\right] \subseteq R^{\prime}[\lambda]$, so we may apply Lemma 5 to conclude $\left[R_{\lambda} M_{0}\right]=0$. Hence, $I=M_{0}$ is an ideal of $L$ which has no intersection with $Z(L)$ since $\operatorname{Ker}\left[v_{(0)} \cdot\right] \cap M_{0}=0$.

Case 2. Assume $R^{\prime}$ is a nontrivial $L_{1}$-module. Then by Proposition 1 there exists an ideal $M_{0}$ of $L_{0} \ltimes R^{\prime}$ such that $\left[R_{(\lambda)}^{\prime} M_{0}\right]=0$. As an $L_{1}$ module, $M_{0}$ is 
isomorphic to $M_{\alpha, \Delta, U}$, where $U$ is either 1-dimensional or an irreducible $\mathfrak{g}$-module. We are going to prove that the ideal generated by $M_{0}$ in the entire algebra $L$ has trivial intersection with its center.

Consider $A=R / R^{\prime}$ as a conformal module over $\mathrm{Vir}=H v \subseteq L_{1}$ with respect to the induced regular action. By Lemma 4, there exists a sequence of conformal Vir-modules $0=A_{-1} \subset A_{0} \subset \cdots \subset A_{n}=A$, where $A_{k} / A_{k-1}$ is either isomorphic to $M_{\alpha_{k}, \Delta_{k}}$ or trivial. Define an index $I(k), k=0, \ldots, n$, in the following way: $I(k)=1$ if $A_{k} / A_{k-1} \simeq M_{\alpha_{k}, \Delta_{k}}$ and $I(k)=0$ if $A_{k} / A_{k-1}$ is trivial (either torsionfree or coincides with its torsion).

Suppose $\bar{a}_{k}, k=0, \ldots, n$, are the generators of $A_{k} / A_{k-1}$ over $H$, and choose the corresponding pre-images $a_{k} \in R$. Then the set $\left\{a_{k}+R^{\prime} \mid k=0, \ldots, n\right\}$ generates $R / R^{\prime}$ over $H$, and

$$
D_{0} a_{k} \in I(k)\left(\partial+\alpha_{k}\right) a_{k}+\sum_{0 \leq i<k} f_{i k}(\partial) a_{i}+R^{\prime},
$$

where $D_{0}=\left[v_{(0)} \cdot\right]$ (we do not define what is $\alpha_{k}$ when $I(k)=0$ ).

Denote

$$
\begin{aligned}
& w_{\alpha_{1,0}, \ldots, \alpha_{k_{0}, 0}, \ldots, \alpha_{1, n}, \ldots, \alpha_{k_{n}, n}}^{k_{0}, \ldots, k_{k}} \\
& \quad=\left[a_{n\left(\alpha_{1, n}\right)} \ldots\left[a_{n\left(\alpha_{k_{n}, n}\right)}\left[a_{n-1\left(\alpha_{1, n-1}\right)} \ldots\left[a_{0\left(\alpha_{1,0}\right)} \ldots\left[a_{0\left(\alpha_{k_{0}, 0}\right)} u\right] \ldots\right]\right]\right],\right.
\end{aligned}
$$

where $u \in U, \alpha_{l, i} \in \mathbb{k}, k_{i} \geq 0$. Let $W\left(a_{0}, \ldots, a_{n}\right) \subset R$ be the set of all $w=$ $w_{\alpha_{1}, 0, \ldots, \alpha_{k_{0}, 0}, \ldots, \alpha_{1, n}, \ldots, \alpha_{k_{n}, n}}^{k_{0}, \ldots, k_{n}}$. It is clear that the $H$-linear span $I$ of all elements from $W\left(a_{0}, \ldots, a_{n}\right)$ is the ideal in $L$ generated by $M_{0}$. Indeed, since $R / R^{\prime}$ is an Abelian Lie conformal algebra and $\left[R_{(\lambda)}^{\prime} u\right]=0$, we have $\left[a_{k(\alpha)} w\right] \in I$ for all $w \in I$ (one may re-arrange the operators $\left[a_{i\left(\alpha_{l, i}\right)} \cdot\right]$ in the desired way). Obviously, $I$ is closed under the multiplication with $L_{i}$ for $i=1, \ldots, s$, but, in general, $\left[L_{i(\lambda)} I\right] \neq 0$ in contrast to Proposition [1.

Define the weight wt $w$ of an expression $w$ of the form (6) as the $(n+2)$-tuple $\left(k_{0}+\cdots+k_{n}, k_{n}, \ldots, k_{0}\right)$, and let the weights be the lexicographically ordered.

Assume $Z(L) \cap I \neq 0$. For every $z \in Z(L) \cap I, z \neq 0$, there exists its presentation

$$
z=h_{1}(\partial) w_{1}+\cdots+h_{m}(\partial) w_{m}, \quad h_{i} \neq 0, \quad w_{m} \in W\left(a_{0}, \ldots, a_{n}\right) ;
$$

such that $\max _{i}$ wt $w_{i}$ is minimal among all presentations of $z$ in the form (7). Denote such a weight by wt $z$. Then, consider those presentations of $z$ in the form (77) with $\max _{i}$ wt $w_{i}=$ wt $z$ and choose one with minimal number $r$ of $w_{i}$ s with wt $w_{i}=$ wt $z$ (say, wt $w_{1}=\cdots=$ wt $w_{r}=$ wt $z$, wt $w_{i}<$ wt $z$ for $i=r+1, \ldots, m$ ). Denote this number $r$ by $\operatorname{deg} z$. Both wt $z$ and $\operatorname{deg} z$ are well-defined: They depend only in $z \in Z(L) \cap I, z \neq 0$.

Straightforward computation shows

$$
D_{0} w=(\partial+\alpha(w)) w+z^{\prime}, \quad \alpha(w) \in \mathbb{k}, \text { wt } z^{\prime}<\operatorname{wt} w
$$

for $w \in W\left(a_{0}, \ldots, a_{n}\right)$.

We may choose $0 \neq z_{0} \in Z(L) \cap I$ such that: (1) wt $z_{0}$ is minimal among all $z \in Z(L) \cap I, z \neq 0$; (2) $r=\operatorname{deg} z_{0}$ is minimal possible among all $z$ with minimal wt $z$.

Then

$$
z_{0}=h_{1}(\partial) w_{1}+\cdots+h_{r}(\partial) w_{r}+z^{\prime}
$$


where wt $w_{1}=\cdots=$ wt $w_{r}=$ wt $z_{0}$ and wt $z^{\prime}<$ wt $z_{0}$. But (8) implies

$$
0=D_{0} z_{0}=h_{1}(\partial)\left(\partial+\alpha\left(w_{1}\right)\right) w_{1}+\cdots+h_{r}(\partial)\left(\partial+\alpha\left(w_{r}\right)\right) w_{r}+z^{\prime \prime},
$$

where wt $z^{\prime \prime}<$ wt $z_{0}$. Hence,

$$
\left(\partial+\alpha\left(w_{1}\right)\right) z_{0} \in(Z(L) \cap I) \backslash\{0\}
$$

has either smaller weight or smaller degree than $z_{0}$. The contradiction obtained proves $I \cap Z(L)=0$.

Theorem 3. Let $L$ be a finite torsion-free conformal Lie algebra with a splitting solvable radical $R$. Then $L$ has a finite faithful conformal representation.

Proof. Suppose $L$ is a counterexample of minimal rank over $H$. Then $Z(L) \neq 0$ : Otherwise, the regular representation is faithful.

By Theorem [1ii), $L_{0}=L_{1} \oplus \cdots \oplus L_{k} \oplus L_{k+1} \oplus \cdots \oplus L_{s}$, where $L_{i}$ are either Cur $\mathfrak{g}$ or Vir or Vir $\ltimes$ Cur $\mathfrak{g}$.

Let $L_{1}, \ldots, L_{k}$ be as in Lemma2 (contain Virasoro element), and let $L_{k+1}, \ldots, L_{s}$ be isomorphic to current conformal algebras. The case $k=0$ was considered in [12, so assume $k \geq 1$, i.e., $L_{0}$ contains Virasoro elements.

The radical $R$ is a conformal module over $L_{i}$ for every $i=1, \ldots, s$. If $R$ is trivial over all $L_{1}, \ldots, L_{k}$ then $L$ can be presented as $L^{\prime} \oplus L^{\prime \prime}$, where

$$
L^{\prime}=L_{1} \oplus \cdots \oplus L_{k}, \quad L^{\prime \prime}=\left(L_{k+1} \oplus \cdots \oplus L_{s}\right) \ltimes R .
$$

Here $L^{\prime}$ is semisimple, $L^{\prime \prime}$ is of the kind considered in 12. Since both $L^{\prime}$ and $L^{\prime \prime}$ have finite faithful conformal representations, so is $L$. Therefore, we may assume $R$ is a nontrivial $L_{1}$-module.

Propositions 1 and2 imply the existence of an ideal $I$ of $L$ such that $I \cap Z(L)=0$. Consider the set $\hat{I}=\{a \in L \mid h(\partial) a \in I$ for some $h \in H\} \supseteq I$. Since $\left[L_{(\lambda)} \hat{I}\right] \subseteq I[\lambda]$, this is also an ideal in $L$. Moreover, if $I \cap Z(L)=0$ then $\hat{I} \cap Z(L)=0$ (recall that the algebra $L$ is torsion-free). Both $L / Z(L)$ and $L / \hat{I}$ are torsion-free finite Lie conformal algebras of smaller rank than $L$, thus have finite faithful representations. The direct sum of these representations would be a faithful finite representation of $L$.

\section{ACKNOWLEDGEMENTS}

I am grateful to Ivan Shestakov for communicating the reference [14, and to the IME USP (Brazil) where the major part of this work has been performed.

\section{REFERENCES}

[1] B. Bakalov, A. D'Andrea, V. G. Kac, Theory of finite pseudoalgebras, Adv. Math. 162 (2001) no. $1,1-140$.

[2] B. Bakalov, V. G. Kac, A. A. Voronov, Cohomology of conformal algebras, Commun. Math. Phys. 200 (1999) 561-598.

[3] A. A. Beilinson, V. G. Drinfeld, Chiral algebras, Amer. Math. Soc. Colloquium Publications 51, AMS, Providence, RI, 2004.

[4] S.-J. Cheng, V. G. Kac, Conformal modules, Asian J. Math. 1 (1997) 181-193.

[5] S.-J. Cheng, V. G. Kac, M. Wakomoto, Extensions of conformal modules, in Topological field theory, primitive forms and related topics (Kyoto, 1996), 79-129, Progr. Math., 160, Birkhäuser, Boston, MA, 1998.

[6] A. D'Andrea, V. G. Kac, Structure theory of finite conformal algebras, Sel. Math., New Ser. 4 (1998) 377-418. 
[7] A. De Sole, V. G. Kac, Lie conformal algebra cohomology and the variational complex, Commun. Math. Phys. 292 (2009) 667-719.

[8] E. Frenkel, D. Ben-Zvi, Vertex algebras and algebraic curves, Mathematical Surveys and Monograps 88, AMS, Providence, RI, 2001.

[9] N. Jacobson, Lie algebras, John Wiley and Sons, New York - London, 1962.

[10] V. G. Kac, Vertex algebras for beginners, second ed., University Lecture Series 10, AMS, Providence, RI, 1998.

[11] P. S. Kolesnikov, Varieties of dialgebras and conformal algebras, Sib. Math. J. 49 (2008) 257-272.

[12] P. S. Kolesnikov, On finite representations of conformal algebras, J. Algebra 331 (2011) 169193.

[13] J.-L. Loday, Dialgebras, in Dialgebras and related operads, J.-L. Loday et al, eds, SpringerVerl., Berlin, 2001, Lectures Notes in Math., vol. 1763, pp. 1-61.

[14] Yu. A. Neretin, A construction of finite-dimensional faithful representation of Lie algebra, Rend. Circ. Mat. Palermo (2) Suppl. No. 71 (2003), 159-161.

[15] C. Procesi, Lie groups. An approach through invariants and representations, Springer, New York, 2007.

[16] M. Roitman, Universal enveloping conformal algebras, Sel. Math., New Ser. 6 (2000), no. 3, 319-345.

[17] M. Roitman, On embedding of Lie conformal algebras into associative conformal algebras, J. Lie Theory 15 (2005) no. 2, 575-588.

Sobolev Institute of Mathematics, Novosibirsk, Russia 